\title{
GSP03
}

\section{Horizontal Well Placement and Segmentation for Completion at M1 Sandstone, Oriente Basin, Ecuador}

\author{
E. Rojas* (Schlumberger), G. Cuadros (Schlumberger), R. Villalva \\ (Schlumberger), R. Chang (Repsol-YPF Ecuador), N. Orellana (Repsol-YPF \\ Ecuador) \& R. Penaranda (Repsol-YPF Ecuador)
}

\section{SUMMARY}

Geosteering application in a challenging geological scenario, where a $1000 \mathrm{ft}$ horizontal section was placed into water driven reservoir, completing the well with inflow control device technology in segmented intervals determined based on petrophysical interpretation and facies characterization from logging while drilling measurements. 
Horizontal drilling in Ecuador is often challenging due to high geological uncertainty and very active oil/water contacts rising as the field is produced. Consequently, the targets for horizontal well placement become narrower as reservoirs are depleted. Conventional geosteering techniques normally fail to detect reservoir boundaries unless the well has actually crossed them, which increases the chance of wells placed close to water contacts while at the same time leaving important attic oil reserves un-drained.

In late 2006, the joint work between Block 16 operator and leader service company resulted in the application of deep directional resistivity technology for the first time in Ecuador and to date it has successfully been used in more than 20 wells improving reservoir geology interpretation by integrating bed boundary detection capability, real-time gamma ray images for near borehole information and cuttings samples analysis. Successful results have reduced the need for pilot holes to the minimum and have allowed operator to target formations never drilled before in Ecuador.

Horizontal wells in Block 16 have historically been completed with screens in approximately $1000 \mathrm{ft}$ of horizontal section. They rapidly reach high water cuts (BSW $~ 95 \%$ ) in a short period of time (3 months or even less) due to active aquifer. This production behavior led the operator to implement techniques to control water production and/or enhance oil recovery. In late 2009, a horizontal well was placed into the M1 sandstone reservoir and completed with non conventional architecture in segmented intervals, including meshes, Inflow Control Devices (ICD) and packers to effectively produce all the exposed section.

Geosteering of this well was performed using deep directional resistivity measurements and the interpretation of dips from real-time gamma ray images, while evaluating reservoir properties with nuclear tool measurements and cuttings. The trajectory was deviated from plan to follow the structure and target higher resistivity zones. At a critical point in the well, inversion indicated the approach of a more resistive layer and the well was geosteered to enter a $13 \mathrm{ft}$ thick sand. Later on, intersected a high dipping kaolinitic sand channel and decided to cross it laterally until entered another sand body, which presented improved resistivities. Trajectory was kept within this prolific zone and additional $114 \mathrm{ft}$ were drilled as compared to the original plan (Figure 1).

Real-time measurements and improved interpretation allowed the identification of reservoir heterogeneities, structural tendencies and construction of a robust petrophysical model. Conventional $\log$ analysis and neural networks were used for petrophysical characterization and determination of segmented intervals with corresponding depths to complete the well with meshes combined with packers and ICD's. The well started producing 2377 BFPD, 2220 BOPD, BSW $=7 \%$ and after 6 months it has produced $258 \mathrm{MBO}$. Current flow is $6064 \mathrm{BFPD}, 1110 \mathrm{BOPD}, \mathrm{BSW}=82 \%$.

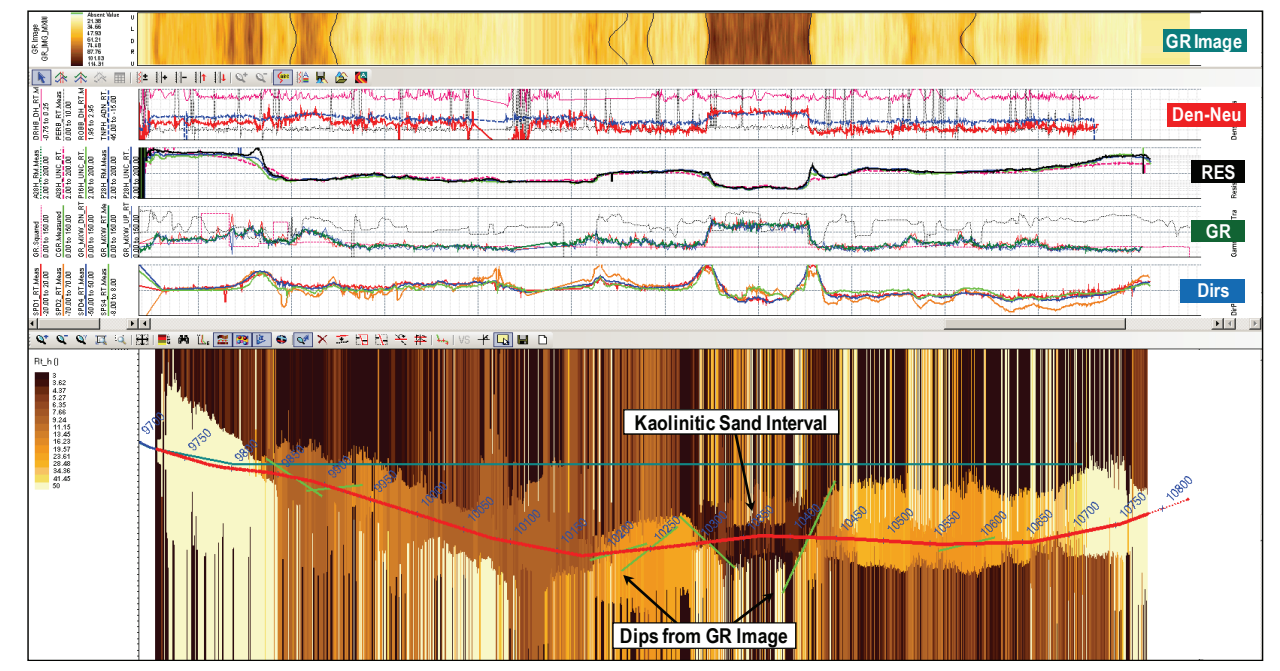

Figure 1 Well geosteered in the M1 sandstone. Inversion of deep directional measurements and log data denote reservoir heterogeneity and provides better determination of the most promising intervals for completion. 